\title{
Displaying Vehicle Driving Mode : Effects on Pedestrian Behavior and Perceived Safety
}

Joisten, Philip; Alexandi, Emanuel; Drews, Robin et al.

(2019)

DOI (TUprints): $\quad$ https://doi.org/10.25534/tuprints-00013460

Lizenz:

lediglich die vom Gesetz vorgesehenen Nutzungsrechte gemäß UrhG

Publikationstyp: Konferenzveröffentlichung

Fachbereich: $\quad 16$ Fachbereich Maschinenbau

Quelle des Originals: https://tuprints.ulb.tu-darmstadt.de/13460 
This is a pre-copyedited version of a contribution published in Human Systems Engineering and Design II. Proceedings of the 2nd International Conference on Human Systems Engineering and Design (IHSED2019): Future Trends and Applications, September 16-18, 2019, Universität der Bundeswehr München, Munich, Germany, published by Springer, Cham. The definitive authenticated version is available online via http://dx.doi.org/10.1007/978-3-030-27928-8_38

\title{
Displaying Vehicle Driving Mode - Effects on Pedestrian Behavior and Perceived Safety
}

\author{
Philip Joisten ${ }^{1}$, Emanuel Alexandi ${ }^{1}$, Robin Drews ${ }^{1}$, Liane Klassen ${ }^{1}$, \\ Patrick Petersohn ${ }^{1}$, Alexander Pick ${ }^{1}$, Sarah Schwindt ${ }^{1}$ and Bettina Abendroth ${ }^{1}$ \\ ${ }^{1}$ Institute of Ergonomics \& Human Factors, Technische Universität Darmstadt, Otto- \\ Berndt-Straße 2, 64287 Darmstadt, Germany \\ \{p.joisten, abendroth\}@iad.tu-darmstadt.de; \{emanuel.alexandi, robin_christian.drews, li- \\ ane.klassen, patrick.petersohn, alexander_klaus_peter.pick, sarah_selina.schwindt\}@stud.tu- \\ darmstadt.de
}

\begin{abstract}
The type and amount of information pedestrians should receive while interacting with an autonomous vehicle (AV) remains an unsolved challenge. The information about the vehicle driving mode could help pedestrians to develop the right expectations regarding further actions. The aim of this study is to investigate how the information about the vehicle driving mode affects pedestrian crossing behavior and perceived safety. A controlled field experiment using a Wizard-of$\mathrm{Oz}$ approach to simulate a driverless vehicle was conducted. 28 participants experienced a driverless and a human-operated vehicle from the perspective of a pedestrian. The vehicle was equipped with an external human machine interface (eHMI) that displayed the driving mode of the vehicle (driverless vs. human-operated). The results show that the crossing behavior, measured by critical gap acceptance, and the subjective reporting of perceived safety did not differ statistically significantly between the driverless and the human-operated driving condition.
\end{abstract}

Keywords: Vehicle Driving Mode $\cdot$ Automation Status $\cdot$ Pedestrian Behavior Perceived Safety $\cdot$ Human Machine Interface $\cdot$ Human Machine Interaction

\section{$1 \quad$ Introduction and Research Question}

Today's road traffic system is constituted by a variety of different forms of implicit (e.g. trajectories) and explicit communication (e.g. gestures) between road users. In order to integrate in mixed traffic environments, autonomous vehicles (AVs) must have the capability to communicate different kinds of information to their environment. The vehicle driving mode is one information category that could help pedestrians to develop the right expectations regarding further actions of the AV $[1,2]$. To provide this information external human machine interfaces (eHMIs) are currently under development and evaluation $[3,4]$. This should contribute to safe, efficient and comfortable interactions between AVs and other road users. Unambiguous communication between the vehicle and other road user is particularly necessary when the vehicle is driving without 
a driver (for instance driverless vehicles). Therefore, the aim of this research is to investigate which effects the display of the driving mode of a driverless vehicle have on the crossing decisions of pedestrians and compare those to ones made interacting with a human-operated vehicle.

Changes in the road transport system and their effects on safety have been studied under the theoretical framework of behavioral adaptation. Behavioral adaptation describes 'those behaviours which may occur following the introduction of changes to the road-vehicle-user system and which were not intended by the initiators of the change' $[5$, p. 23]. The theoretical framework of behavioral adaptation is promising to study the effects of introducing eHMIs on the behavior of pedestrians. Based on the research aim, following research question is formulated: Does displaying the vehicle driving mode of a driverless vehicle result in behavioral adaptation of pedestrians?

Theory suggests that the monitoring and attunement of risk plays a major role in the formation of behavioral adaptation [6]. If a vehicle with a given system (e.g. an eHMI) provides an improved feeling of control compared to a vehicle without the system, the assumed risk reduction might be compensated by a change in pedestrian behavior [7]. An eHMI can explicitly communicate the vehicle driving mode to pedestrians, therefore provide an improved feedback and feeling of control. Based on the research question and the brief insight into behavioral adaptation theory, two hypotheses are formulated:

- H1: An eHMI displaying the vehicle driving mode of a driverless vehicle increases the subjective feeling of perceived safety of pedestrians in comparison to a humanoperated vehicle.

- H2: An eHMI displaying the vehicle driving mode of a driverless vehicle reduces the critical gap acceptance of pedestrians in comparison to a human-operated vehicle.

\section{$2 \quad$ Method and Materials}

\subsection{Research Design, Procedure and Materials}

To test the hypotheses a controlled field experiment was conducted on a test ground, a former airfield near Darmstadt, Germany. Participants were invited to experience a driverless and a human-operated vehicle from the perspective of a pedestrian. The scenario used in the experiment was an un-signalized crossing of a straight road with no obstructions of visibility and no other traffic participants present. Participants stood at the side of the road at the distance of 2.75 meters from the middle of the traffic lane. The vehicle drove past the participants with a constant speed of $30 \mathrm{~km} / \mathrm{h}$ in each trial.

The procedure of the within-subject design study was as follows: In the first trial the vehicle drove past the participants in the driverless and the human-operated driving condition (in permuted order). The participants stood with their back to the road and turned towards the vehicle when it was in a distance of 100 meters. The task of the participants in the first trial was to observe the passing vehicle. After each passing of the vehicle the participants had to indicate which driving mode they experienced (manual, automated, driverless or other) and what caused them to make their decision. A 
written explanation of different terms related to automated driving was given to the participants before the experiment started.

In the second trial the participants experienced the driverless and the human-operated driving condition again in permuted order to avoid sequencing effects. Participants were informed about the different vehicle conditions and instructed to turn to the vehicle when it was 100 meters away. Their task was to cross the road in front of the oncoming vehicle. For safety reasons the participants never actually crossed or stepped on the road in front of the vehicle. The critical gap acceptance and perceived safety were measured after each passing of the vehicle (see chapter 2.3).

To simulate a driverless, fully automated vehicle (SAE Level 5), the Wizard-of-Oz approach "Ghostdriver" was used [8]. A seat-costume covers the driver so that outside road users cannot see him. In addition, a prototype eHMI was developed to explicitly communicate the vehicle driving mode to other road users (see Fig. 1). The eHMI (width: $420 \mathrm{~mm}$, height: $300 \mathrm{~mm}$ ) is positioned in front of the radiator grill. A translucent plate with an engraved symbol is placed behind a light which can be switched on and off. The color of the eHMI is turquoise.
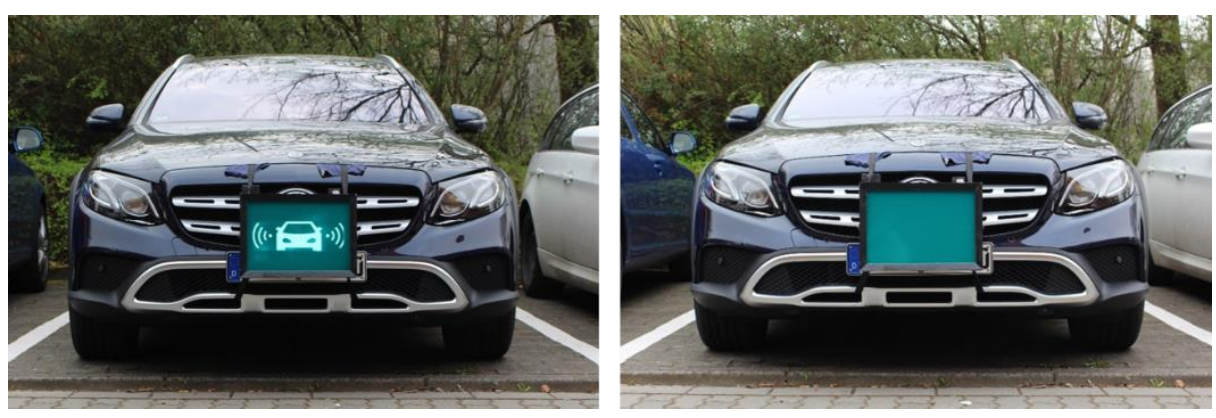

Fig. 1. eHMI used to explicitly communicate the vehicle driving mode in the study. Left: activated eHMI with symbol representing the autonomous driving mode; Right: deactivated eHMI without symbol.

\subsection{Independent Variable: Vehicle Driving Mode}

As independent variable the vehicle driving mode was manipulated. The conditions were driverless (see Fig. 1 left) and human-operated vehicle (see Fig. 1 right). In the driverless condition the driver was covered by the seat-costume whereas he was well visible in the manual driving condition. The eHMI was visible in both conditions but only switched on in the driverless condition. In addition, a symbol representing an autonomous car was visible in the driverless condition. The symbol was based on a research on the labeling of autonomous systems.

\subsection{Dependent Variable: Pedestrian Behavior and Perceived Safety}

The dependent variable pedestrian behavior was measured by the critical gap acceptance which indicates the last moment the pedestrian is willing to cross the road in front of the vehicle [2]. Participants were given a stopwatch which they were instructed 
to start at the last moment they are willing to cross the road in front of the vehicle. The stopwatch was then handed over to a trained researcher who stopped the stopwatch when the vehicle reached a defined point on the road ( 25 meters behind the participant).

To assess changes in perceived safety a questionnaire was immediately filled out by the participants after they experienced the driverless and the human-operated vehicle in trail two. Participants indicated their perceived safety on a 5-point scale (-2 "I feel unsafe", 0 - "Indifferent", 2 "I feel safe").

\subsection{Participants}

28 participants $(21 \%$ female, $79 \%$ male, mean age $=25.2$ years, $\mathrm{SD}=2.86$ years $)$ took part in the study. $75 \%$ of the participants stated to participate in road traffic several times a day as a pedestrian. $18 \%$ stated to participate daily and $7 \%$ to participate approx. every second day in road traffic as a pedestrian.

\section{Results}

\subsection{Identification of Vehicle Driving Mode}

In the first trail participants experienced both (driverless and human-operated) driving conditions and indicated after each passing of the vehicle which driving mode they experienced. The answers of the participants are summarized in Table 1.

Table 1. Reported driving conditions by the participants in the first trail.

\begin{tabular}{lll}
\hline Answers & $\begin{array}{l}\text { Driverless vehicle } \\
\text { condition }(\mathbf{N}=\mathbf{2 7})\end{array}$ & $\begin{array}{l}\text { Human-operated vehicle } \\
\text { condition }(\mathbf{N = 2 8})\end{array}$ \\
\hline Human-operated & - & $20(71 \%)$ \\
Autonomous & $2(7 \%)$ & $8(29 \%)$ \\
Driverless & $25(93 \%)$ & - \\
\hline
\end{tabular}

Participants were asked what caused them to make their decision regarding the vehicle driving mode. In the driverless vehicle condition the main reason reported was the empty driver seat ( $89 \%$ of the answers) followed by the eHMI $(11 \%)$. In the humanoperated driving condition, the main reasons reported was the visible driver.

\subsection{Changes in Pedestrian Behavior}

In the second trial, the behavioral change of the participants, measured by critical gap acceptance, was determined. In the driverless vehicle condition (Wizard-of-Oz seatcostume and activated eHMI) the mean critical gap acceptance was $5.3 \mathrm{~s}(\mathrm{SD}=1.6 \mathrm{~s})$. The mean critical gap acceptance in the human-operated driving condition (visible driver and deactivated eHMI) was $5.1 \mathrm{~s}(\mathrm{SD}=1.4 \mathrm{~s})$. The critical gap acceptance did not differ statistically significantly between the two conditions (dependent t-test, $|\mathrm{T}|=$ 1.192 , df $=27, \mathrm{p}=.244$ ). 


\subsection{Changes in Perceived Safety}

To assess changes in perceived safety a questionnaire was presented after the participants experienced the driverless and the human-operated vehicle in the second trial. Perceived safety was indicated on a 5-point scale (-2 - "I feel unsafe", 0 - "Indifferent", 2 - "I feel safe"). The results between the driverless vehicle condition (mean $=0.47$, $\mathrm{SD}=0.92)$ and human-operated vehicle condition (mean $=0.71, \mathrm{SD}=0.98)$ did not differ statistically significantly (dependent t-test, $|\mathrm{T}|=1.022, \mathrm{df}=27, \mathrm{p}=.316$ ).

With regard to the eHMI participants were also asked how the eHMI influenced their feelings of safety. $57 \%$ of participants reported a positive effect of the eHMI on their perceived safety. $32 \%$ reported no effect and $11 \%$ a negative effect.

\section{Discussion}

The present study investigates the effects of communicating the vehicle driving mode through an eHMI on the crossing decision of pedestrians. The results show that neither the subjective ratings of perceived safety nor the measured critical gap acceptance differ statistically significantly between the driverless and the human-operated vehicle condition. In line with related research [see 2], hypotheses 1 and 2 are not supported.

Communication of vehicle driving mode could foster system transparency and thus help pedestrians to attune to the right expectations regarding their and the vehicle's further actions [1,9]. The question of labelling the automation status might become increasingly important the more unclear it is who is driving the vehicle (human vs. automation). Learnt behavior plays a dominant role in the first interaction with AVs [2] which might suppress short-term effects of behavioral adaptation.

The identification of vehicle driving mode communicated via an eHMI must be unambiguous. The mere presence of the eHMI led to mode confusion among several participants. To prevent this, further development of eHMIs should explicitly consider possible effects of mode confusion caused by displaying (or not displaying) the automation status of the vehicle.

The influence of the design of the eHMI on the present study results must be discussed critically. Although the chosen symbol representing the automation status of the vehicle was seen as positive by participants it was not unambiguous for all. A possible solution for unambiguous communication could be standardized symbolism [3].

No changes in pedestrian behavior measured by critical gap acceptance were found in the present study. To draw the right conclusion, the method to obtain the measure of critical gap acceptance should be critically discussed. The method used via time-taking at defined points in the infrastructure is highly dependent on human influence (and human error). To minimize possible errors all researchers involved in the study completed a training. In future studies more reliable approaches to measure critical gap acceptance should be used.

Prior research shows that pedestrians perceive autonomous car traffic less risky than human-operated car traffic [10]. In the present study, the driverless vehicle was not perceived as more or less safe than the human-operated vehicle. Post-hoc interviews showed that no participant discovered the Wizard-of-Oz seat-costume. In contrast to survey studies [e.g. 10], participants experienced a real "driverless" vehicle. 
Although no statistically significant effect of perceived safety between the driverless and the human-operated vehicle condition was found, the eHMI had a slightly positive effect on reported feelings of safety in post-hoc interviews. To distinguish the effect of the eHMI and driverless vehicle on perceived safety a comparison between a driverless vehicle with and without eHMI has to be carried out. This was beyond the scope of the present study.

\section{Conclusion}

This study gives first insights into the effects of displaying the vehicle automation status on pedestrian behavior and perceived safety. Overall, the eHMI displaying the vehicle automation status did not influence pedestrian behavior and perceived safety. Further evaluation of the eHMI design is necessary for an unambiguous interaction between $\mathrm{AV}$ and pedestrian.

The theoretical framework of behavioral adaptation seems promising for the investigation of the effects of eHMIs on pedestrian safety. Pedestrians' behavioral adaptation beyond the first interaction with an AV will be investigated in further studies.

Acknowledgments. The present study was supported by the project@CITY-AF which receives funding from the German Federal Ministry of Economy and Energy (BMWi).

\section{References}

1. Schieben, A., Wilbrink, M., Kettwich, C., Madigan, R., Louw, T., Merat, N.: Designing the interaction of automated vehicles with other traffic participants: design considerations based on human needs and expectations. Cogn Technol Work 21, 69--85 (2019)

2. Rodríguez Palmeiro, A., Kint, S van der., Vissers, L., Farah, H., Winter, J.C.F. de, Hagenzieker, M.: Interaction between pedestrians and automated vehicles: A Wizard of Oz experiment. Transp Res Part F Traffic Psychol Behav 58, 1005--1020 (2018)

3. Ackermann, C., Beggiato, M., Schubert, S., Krems, J.F.: An experimental study to investigate design and assessment criteria: What is important for communication between pedestrians and automated vehicles? Appl Ergon 75, 272--282 (2019)

4. Clerq, K. de, Dietrich, A., Núñez Valesco, J.P., Winter, J. de, Happee, R.: External HumanMachine Interfaces on Automated Vehicles: Effects on Pedestrians Crossing Decisions. Hum Factors (2019)

5. OECD: Behavioural adaptation to changes in the road transport system. Paris (1990)

6. Jiang, C., Underwood, G., Horwarth, C.I.: Towards a theoretical model for behavioural adaptations to changes in the road transport system. Transport Rev 12, 253--263 (1992)

7. Vaa, T.: Psychology of Behavioural Adaptation. In: Rudin-Brown, C.M., Jamson, S.L. (eds.) Behavioural Adaptation and Road Safety, pp. 207--226. CRC Press, Boca Raton, FL (2013)

8. Joisten, P., Müller, A., Walter, J., Abendroth, B., Bruder, R.: Neue Ansätze der Human Factors Forschung im Zeitalter des Hochautomatisierten Fahrens. In. Bruder, R., Winner, H. (eds.) Hands off, Human Factors off? Welche Rolle spielen Human Factors in der Fahrzeugautomation? 9. Darmstädter Kolloquium, pp. 69--88. Darmstadt (2019)

9. Selkowitz, A.R., Lakhmani, S.G., Chen, J.Y.C.: Using agent transparency to support situation awareness of the Autonomous Squad Member. Cogn Syst Res 46, 13--25 (2017)

10. Hulse, L.M., Xie, H., Galea, E.R.: Perceptions of autonomous vehicles: Relationships with road users, risks, gender and age. Saf Sci 102, 1--13 (2018) 